\title{
Analysis and fit of stellar spectra using a mega-database of CMFGEN models
}

\author{
Celia Fierro-Santillán ${ }^{1}$, Janos Zsargó2 ${ }^{2}$, Jaime Klapp ${ }^{1,3}$, \\ Santiago Alfredo Díaz-Azuara ${ }^{4}$, Anabel Arrieta ${ }^{5}$ and Lorena Arias ${ }^{5}$ \\ ${ }^{1}$ Centro de Matemática Aplicada y Cómputo de Alto Rendimiento ABACUS-CINVESTAV, \\ Carretera México-Toluca km 38.5, 52740 Estado de México, México. \\ email: celia.fierro.estrellas@gmail.com \\ ${ }^{2}$ Escuela Superior de Física y Matemáticas, Instituto Politécnico Nacional, México. \\ ${ }^{3}$ Instituto Nacional de Investigaciones Nucleares, México. \\ ${ }^{4}$ Instituto de Astronomía, Universidad Nacional Autónoma de México, México. \\ ${ }^{5}$ Universidad Iberoamericana, México.
}

\begin{abstract}
We present a tool for analysis and fit of stellar spectra using a mega database of 15,000 atmosphere models for OB stars. We have developed software tools, which allow us to find the model that best fits to an observed spectrum, comparing equivalent widths and line ratios in the observed spectrum with all models of the database. We use the $\mathrm{H}_{\alpha}, \mathrm{H}_{\beta}, \mathrm{H}_{\gamma}$, and $\mathrm{H}_{\delta}$ lines as criterion of stellar gravity and ratios of He II $\lambda 4541 / \mathrm{He}$ I $\lambda 4471$, He II $\lambda 4200 /(\mathrm{He}$ I+He II $\lambda 4026)$, He II $\lambda 4541 / \mathrm{He}$ I $\lambda 4387$, and He II $\lambda 4200 / \mathrm{He}$ I $\lambda 4144$ as criterion of $T_{\text {eff }}$.
\end{abstract}

Keywords. stars: atmospheres, stars: abundances, stars: rotation, stars: winds

\section{Database and results}

The models were calculated with the CMFGEN code (Hillier, D. J. \& Miller, D., 1998); database is a matrix arrangement in a 6-dimensional space: surface temperature $\left(T_{\text {eff }}\right)$, luminosity $(L)$, terminal velocity of the wind $\left(V_{\infty}\right)$, beta exponent of the wind velocity law $(\beta)$, clumping filling factor $\left(F_{c l}\right)$, and metallicity $(Z)$. The synthetic spectra in

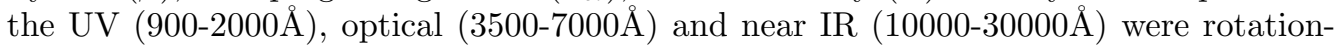
ally broaden using ROTIN3 (Hubeny \& Lanz, 1995) by covering the range between 10 and $350, \mathrm{~km} / \mathrm{s}$ with steps of $10 \mathrm{~km} / \mathrm{s}$. Models were calculated using the ABACUS I super- computer of Centro de Matemáticas Aplicadas y Cómputo de Alto Rendimiento ABACUS-CINVESTAV, México. Software tools allow us to find in the database the model with the best fit to an observed spectrum in a only minutes.
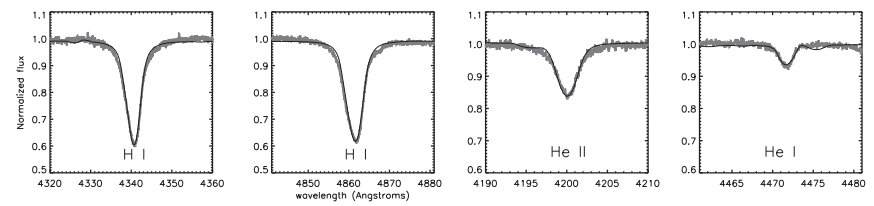

Figure 1. Adjustment example: HD 46223, spectral type O4 V (f) (Sota, A. et al. 2011) observed spectrum from public data of the IACOB project, 2016. Parameters of model: $T_{\text {eff }}=40000 \mathrm{~K}$, $\log L / L_{\odot}=6.05, V_{\infty}=2370 \mathrm{~km} / \mathrm{s}, \beta=1.1, F_{c l}=0.05, Z=Z_{\odot}$

\section{References}

Hillier, D. J. \& Miller, D. 1998, ApJ, 496, 407

Hubeny, I, Lanz, T. 1995, ApJ, 439, 875

Sota, A. et al. 2011, ApJS, 193(2), 24 